\section{Stand und Erfahrungen bei der Umsetzung der EU-Hochwasserrichtlinie}

Die Richtlinie 2007/60/EG wurde vom Europäischen Parlament am 23. Oktober 2007 verabschiedet. Die bundesgesetzliche Verankerung erfolgte im Jahre 2011. Das Heft 5-6/2012 (Juni) der Österreichischen Wasser- und Abfallwirtschaft befasste sich mit den Erfahrungen in der frühen Umsetzungsphase der Richtlinie. Seither wurden die hochwassergefährdeten Bereiche sowie Gebiete mit potenziell signifikantem Hochwasserrisiko (APSFR-Gebiete) ausgewiesen. Ein Instrumentarium für das Hochwassermanagement wurde entwickelt und anhand von einigen Pilotstudien im Hinblick auf seine praktische Anwendbarkeit geprüft.

In diesem Heft wird der aktuelle Stand zur weiteren Umsetzung der Richtlinie anhand von Beiträgen der Bundesverwaltung, der Landesplanung, von Zivilingenieurbüros und der universitären Ebene dargestellt. Sämtliche beteiligten Institutionen arbeiteten in den letzten Jahren bei verschiedenen Pilotprojekten eng zusammen, wodurch ein reger interner fachlicher Austausch initiiert wurde.

Generell hat die Richtlinie das Ziel, einen Rahmen für die Bewertung und das Management von Hochwasserrisiken zu schaffen und dadurch nachteilige Folgen für die menschliche Gesundheit, die Umwelt, das Kulturerbe und wirtschaftliche Tätigkeiten zu vermeiden. Als Grundlage dient die Risikobewertung, für die insgesamt 20 unterschiedliche Risikoindikatoren herangezogen wurden. Als Ergebnis wurden 391 signifikante Risikogebiete bestimmt, was 7,1\% der Gesamtlänge aller untersuchten Gewässer entspricht.

In einem zweiten Schritt wurden für diese Risikogebiete Hochwassergefahrenkarten und Hochwasserrisikokarten erarbeitet. Die Gefahrenkarten zeigen die Überflutungsflächen, Wassertiefen und Fließgeschwindigkeiten für drei unterschiedliche Hochwasserwahrscheinlichkeiten. Im dritten Arbeitsschritt werden Hochwasserrisikomanagementpläne erstellt. Dafür werden Ziele zur Risikoreduktion sowie Umsetzungsmaßnahmen festgelegt. Der Schwerpunkt der Maßnahmen liegt auf Vermeidung, Schutz und Vorsorge. Letztendlich soll im Rahmen eines „integralen Risikomanagements“ durch ein Zusammenwirken von naturnahen, raumplanerischen, bautechnischen, organisatorischen, nicht-baulichen und bewusstseinsbildenden Maßnahmen die Gefährdung von Menschen und die Schadwirkungen von Hochwässern auf ein akzeptables Maß reduziert werden.

Die Hochwasserrichtlinie unterstreicht klar die Bedeutung von nicht-technischen Maßnahmen, wozu u. a. der Erhalt natürlicher Retentionsräume, die Abstimmung der Landnutzung mit den Hochwassergefahren und die Vermeidung eines Risikozuwachses in Restrisikogebieten zählen. Dafür sind Instrumente im Interaktionsbereich von Raumentwicklung und Wasserwirtschaft zu entwickeln und zu erproben. Wichtig, wertvoll und schadensmindernd sind dabei Bewusstseinsbildung, die Kenntnis der persönlichen Gefährdung und das Wissen um die Möglichkeiten zur Eigenvorsorge. Für eine wirksame Vermeidung und Verminderung von Hochwasserrisiken ist es unumgänglich, die laufende wasserwirtschaftliche Entwicklung in Überflutungsgebieten zu beobachten und über neue Evaluierungs- und Prognoseinstrumente zu steuern.

Die Bundesländer entwickelten von März bis September 2014 die Hochwasserrisikomanagementpläne. Diese sind für alle Gebiete mit potenziell signifikantem Hochwasserrisiko auszuarbeiten. Für eine integrative Planung erfordert die Querschnittsmaterie „Hochwasserrisikomanagement“ die Abstimmung aller föderalen Ebenen (Bundes-, Landes- und Regionalebene) sowie die fachübergreifende Vernetzung und Koordinierung aller involvierten Stellen. Die Vorgehensweise erfolgte in den einzelnen Bundesländern unterschiedlich. In der Steiermark und in Kärnten wurde dazu eine detaillierte Methodik des Prozessablaufs entwickelt, der eine intensive und frühzeitige Einbindung der verantwortlichen und berührten Stellen vorsieht.

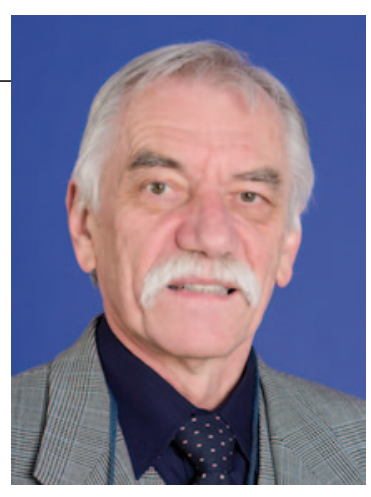

em. o.Univ.-Prof. DI

Dr. Hans Peter Nachtnebel 
Als Beispiel wird die Erstellung des „Hochwasserrisikomanagementplan Gleisdorf“ beschrieben. Einerseits dienen diese Pläne zur lokalen Bewusstseinsbildung über die Hochwassergefahren, andererseits erfolgte im Zuge des Pilotprojektes die Weiterentwicklung und Anpassung eines Maßnahmenkataloges, welcher bereits in zwei zuvor durchgeführten Pilotprojekten erarbeitet wurde. Signifikanter Unterschied zu vorhergehenden Pilotprojekten ist eine in Gleisdorf breitflächig angesiedelte Industrienutzung. Aufbauend auf den Ergebnissen des Hochwassermanagementplanes, der Gefahren- und Risikokarten und anderen vorliegenden Grundlagendaten wurde ein Hochwasseralarmplan für die Stadtgemeinde Gleisdorf als beispielhafte Umsetzung einer Maßnahme erstellt.

Die Beurteilung der Hochwasserschadenspotenzials stellt eine wesentliche Grundlage für die Maßnahmenplanung zur Reduktion bestehender Risiken sowie zur Vermeidung des weiteren Anstieges dar. Dies erfordert eine voraus schauende Schadenspotenzialermittlung, die auf die Erweiterung von Siedlungs- und Industriegebieten in Risikozonen Bedacht nimmt, die die Bevölkerungsentwicklung differenziert beachtet und die mögliche Auswirkungen von Klimaänderungen auf die Abflussentstehung berücksichtigt. Diese Analysen dienen zur frühzeitigen Erkennung bzw. Vermeidung von steigenden Schadenspotenzialen.

em. o.Univ.-Prof. DI Dr. H. P. Nachtnebel

Department Wasser - Atmosphäre - Umwelt

Institut für Wasserwirtschaft, Hydrologie und konstruktiven Wasserbau

Universität für Bodenkultur Wien

Muthgasse 18, 1190 Wien, Österreich

hans_peter.nachtnebel@boku.ac.at 